\title{
Effect of Poly-L-arginine on Intestinal Absorption of Hydrophilic Macromolecules in Rats
}

\author{
Tsutomu Yamaki, ${ }^{a}$ Masaki Uchida, ${ }^{a}$ Yusuke Kuwahara, ${ }^{a}$ Yohei Shimazaki, ${ }^{a}$ Kazuo Ohtake, ${ }^{a}$ \\ Mitsutoshi Kimura, ${ }^{a}$ Hiroyuki Uchida, ${ }^{a}$ Jun Kobayashi, ${ }^{a}$ Masahiko Ogihara, ${ }^{a}$ \\ Yasunori Morimoto, ${ }^{a, b}$ and Hideshi Natsume*,a,b \\ ${ }^{a}$ Faculty of Pharmaceutical Sciences, Josai University; and ${ }^{b}$ Research Institute of TTS Technology, Josai University; \\ 1-1 Keyakidai, Sakado, Saitama 350-0295, Japan. \\ Received November 6, 2012; accepted December 6, 2012; advance publication released online December 15, 2012
}

We have already reported that poly-L-arginine (PLA) remarkably enhanced the in vivo nasal absorption of hydrophilic macromolecules without producing any significant epithelial damage in rats. In the present study, we examined whether PLA could enhance the absorption of a model hydrophilic macromolecule, fluorescein isothiocyanate-dextran (FD-4), across the intestinal mucosa, as well as the nasal mucosa, by an in situ closed-loop method using the rat intestine. PLA was found to enhance the intestinal absorption of FD-4 in a concentration-dependent manner within the concentrations investigated in this study, but segment-specific differences were found to be associated with this effect (ileum $>$ jejunum $>$ duodenum $\geqq$ colon). The factors responsible for the segment-specific differences were also investigated by intestinal absorption studies using aprotinin, a trypsin inhibitor, and an analysis of the expression of occludin, a tight junction protein. In the small intestine, the differences in the effect of PLA on the absorption of FD-4 may be related to the enzymatic degradation of PLA. In the colon, the reduced effect of PLA on the absorption of FD-4 may be related to the smaller surface area for absorption and the higher expression of occludin compared with other segments.

Key words poly-L-arginine; intestinal absorption; enhancer; fluorescein isothiocyanate-dextran (FD-4); occludin; aprotinin

We have already reported that poly-L-arginine (PLA), a polycation, remarkably enhanced the in vivo nasal absorption of hydrophilic macromolecules in rats and increased their in vitro permeation across the rabbit nasal epithelium without producing any significant epithelial damage. ${ }^{1-5)}$ This effect is caused by the opening of tight junctions (TJ) in the epithelial cells. PLA plays an important role in opening the TJ in these cells, and the reaction induced by PLA appears to be reversible. ${ }^{4,6)}$ Therefore, PLA is considered to be promising as an absorption enhancer for transnasal drug delivery. Although some studies have examined whether PLA can also enhance the absorption of hydrophilic macromolecules across the nasal epithelium, conjunctiva, alveolar epithelium, and Caco-2 cell sheets, ${ }^{7-10)}$ there have been no studies looking at PLA $\square \mathrm{s}$ absorption enhancing effect across the intestinal mucosa. The intestinal mucosa is an important tissue particularly for the absorption of orally administered drugs.

The intestinal absorption of hydrophilic macromolecules is well known to be typically very poor. ${ }^{11)}$ The small intestine has an extensive surface area for absorption, but various digestive enzymes exist in the gastrointestinal tract. The property of intestinal mucosa differs from that of the nasal mucosa in some respects. Therefore, in order to improve the absorption of hydrophilic macromolecules, various strategies such as the use of absorption enhancers, protease inhibitors and chemical modification have been examined in the intestinal mucosa as well as the nasal mucosa. ${ }^{12-15)}$

It was recently reported that the TJ barrier function, as indicated by the epithelial resistance, varied in a segment-specific way in the rat intestinal epithelium. The segmental expression of occludin and claudins, which are the major TJ proteins, has also been reported to be related to the barrier function. ${ }^{16}$

The authors declare no conflict of interest.
As a result, segment-specific differences may therefore exist regarding the effect of PLA on the intestinal absorption of hydrophilic macromolecules.

In the present study, we examined whether PLA could enhance the absorption of a model hydrophilic macromolecule, fluorescein isothiocyanate-dextran (FD-4), across the intestinal mucosa by an in situ closed-loop method using the rat intestine. In addition, we also examined whether there were segment-specific differences in the effects of PLA on the intestinal absorption using intestinal segments (duodenum, jejunum, ileum and colon). We then investigated the factors responsible for the segment-specific differences, while focusing on the influences of the enzymes involved in PLA degradation and the expression of occludin, one of the TJ proteins, in each intestinal segment. Namely, intestinal absorption studies using aprotinin, a trypsin inhibitor, and immunoblot assays of the occludin expression level were performed.

\section{MATERIALS AND METHODS}

Materials Fluorescein isothiocyanate-dextran (molecular weight (MW) $3.33 \mathrm{kDa}, \mathrm{FD}-4$ ), poly-L-arginine hydrochloride (MW $44.3 \mathrm{kDa}$, PLA) and aprotinin from the bovine lung were purchased from Sigma-Aldrich Co. (MO, U.S.A.). The BCA protein assay reagent kit and ECL chemiluminescence detection system (SuperSignal West Dura Extended Duration Substrate) were obtained from Pierce Biotechnology, Inc. (IL, U.S.A.). Mouse monoclonal anti-occludin, mouse monoclonal anti- $\beta$-actin and horse-radish peroxidase (HRP)-conjugated goat anti-mouse immunoglobulin $\mathrm{G}(\mathrm{IgG})$ were purchased from Santa Cruz Biotechnology, Inc. (CA, U.S.A.). The protease inhibitor cocktail was obtained from Roche Diagnostics GmbH (Mannheim, Germany).

Animals Male Wistar rats, eight weeks of age, weighing 
250-300g, were obtained from Saitama Experimental Animals Supply Co., Ltd. (Saitama, Japan) and kept on a 12/12 h light/dark cycle with at least seven days of local vivarium acclimatization before experimental use. The rats were fasted for about $16 \mathrm{~h}$ prior to undergoing the experiments. All of the protocols involving animals were approved by the Institutional Animal Care and Use Committee at the University of Josai Life Science Center.

Intravenous (i.v.) Injection Study A FD-4 solution (in $2 \mathrm{mg} / \mathrm{mL}$ saline, $2 \mathrm{mg} / \mathrm{kg}$ ) was injected into the right jugular vein of anesthetized rats $(25 \%$ urethane, $1 \mathrm{~g} / \mathrm{kg}$, intraperitoneally (i.p.)), and then they underwent the same surgical procedure as that used in the intestinal absorption study.

Intestinal Absorption Study An intestinal absorption study was performed using an in situ closed-loop method. ${ }^{17)}$ Briefly, after anesthetizing the rats (weighing 250-260g) with urethane, the intestine was exposed through a midline abdominal incision, an intestinal loop (length: $5 \mathrm{~cm}$ ) was made in segments of the duodenum ( $5 \mathrm{~cm}$ distal from the pylorus), jejunum (between 10 and $15 \mathrm{~cm}$ distal from the pylorus), ileum (between 5 and $10 \mathrm{~cm}$ proximal from the cecum), or the colon $(5 \mathrm{~cm}$ distal from the cecum). The intestinal contents were removed by the slow infusion of saline and air. In the duodenum, the bile duct was cannulated with polyethylene tubing (i.d. $0.28 \mathrm{~mm}$ ) to form a bile fistula. FD-4 solution (in saline, $20 \mathrm{mg} / \mathrm{kg}$ ) with or without different concentrations of PLA, or FD-4 solution $(20 \mathrm{mg} / \mathrm{kg})$ containing $100 \mu \mathrm{g} / \mathrm{mL}$ aprotinin and $0.5 \%$ PLA were introduced into the intestinal loop at $100 \mu \mathrm{L} /$ $\mathrm{cm}$ loop. In previous study, the enhancing effect of PLA on nasal absorption was the almost maximum at the concentration of $0.5 \%$. In addition, the epithelial damage was not observed at $0.5 \%$ PLA. ${ }^{2)}$ Therefore, PLA concentration was used up to $0.5 \%$.

Collection of Plasma Blood samples were collected from the left jugular vein using a heparinized syringe at predetermined times, and then were centrifuged at $20400 \times \boldsymbol{g}$ for $5 \mathrm{~min}$ at $4^{\circ} \mathrm{C}$ to obtain plasma.

Determination of the Plasma FD-4 Concentration The plasma was diluted 100 times with potassium dihydrogenphosphate-sodium borate buffer $(\mathrm{pH}$ 8.5). The fluorescence intensity of FD-4 was determined using a fluorescence spectrofluorometer (RF-5000, Shimadzu, Kyoto) at an excitation wavelength of $495 \mathrm{~nm}$ and an emission wavelength of $515 \mathrm{~nm}$. The limit of determination was $0.04 \mu \mathrm{g} / \mathrm{mL}$.

Data Analysis The plasma data for FD-4 were analyzed by a nonlinear least squares regression program (Algorithm: Damping Gauss-Newton method). ${ }^{18)} C_{\max }$ and $T_{\max }$ were obtained from the drug concentration-time curve, and the area under the plasma concentration-time curves $(A U C)$ were calculated by a moment analysis. The bioavailability (\%) based on the period from $0-4 \mathrm{~h}\left(F_{0-4 \mathrm{~h}}\right)$ was calculated from the amount of absorption obtained by means of a deconvolution method.

Immunoblot Analysis The epithelial cells were detached from the excised segments of the rat intestine using slide glasses. The cells were homogenized in Tris-buffer containing $20 \mathrm{~mm}$ Tris, $5 \mathrm{~mm} \mathrm{MgCl}_{2}, 1 \mathrm{~mm}$ ethylenediaminetetraacetic acid (EDTA), $0.3 \mathrm{~mm}$ ethylene glycol-bis(2-aminoethylether)$N, N, N^{\prime}, N^{\prime}$-tetraacetic acid (EGTA) and a protease inhibitor cocktail, and subsequent passage through a $26 \mathrm{G} \times 1 / 2$ needle. Membrane fractions were obtained by two centrifugation steps $\left(5 \mathrm{~min}\right.$ at $1000 \times \boldsymbol{g}, 30 \mathrm{~min}$ at $43000 \times \boldsymbol{g}, 4^{\circ} \mathrm{C}$ ). The pellets were then re-suspended in Tris-buffer. The protein contents were determined by the bicinchoninic acid (BCA) protein assay. The samples were mixed with equal volumes of Laemmli sample buffer $\left(2 \times\right.$ concentrated) and heated at $95^{\circ} \mathrm{C}$ for $10 \mathrm{~min}$. Proteins $(10 \mu \mathrm{g})$ were then separated by sodium dodecyl sulfate-polyacrylamide gel electrophoresis (SDS-PAGE) and transferred to polyvinylidene difluoride (PVDF) membranes. The membranes were blocked in phosphate buffered saline (PBS)-T (PBS containing 0.1\% Tween 20) with 3\% skim milk. They were subsequently incubated in primary antibody in blocking buffer at $4^{\circ} \mathrm{C}$ overnight, followed by incubation for $1 \mathrm{~h}$ with the secondary antibodies. The chemiluminescence was detected using an LAS-1000 instrument (GE Healthcare, U.K.). The pixel density of occludin was determined using the Image J software program.

Statistical Analysis The statistical analysis was performed using Student's $t$-test. A $p$-value $<0.05$ was considered to be statistically significant.

\section{RESULTS AND DISCUSSION}

Effect of PLA on the Intestinal Absorption of FD-4 Figure 1 shows the plasma concentrations of FD-4 following intra-intestinal administration into the closed-loop in rats. Table 1 summarizes the pharmacokinetic parameters obtained for FD-4. PLA enhanced the absorption of FD-4 in all segments of the intestine. The highest enhancement of FD-4 absorption by PLA was observed in the ileum. The intestinal absorption of FD-4 increased in a PLA concentrationdependent manner. When $0.5 \%$ PLA was co-administered into the ileum, the $F_{0-4 \mathrm{~h}}$ was $23.7 \%$. The order of the $F_{0-4 \mathrm{~h}}$ was ileum $>$ jejunum $>$ duodenum $\geqq$ colon. These results indicate that PLA enhances the intestinal absorption of FD-4 in a concentration-dependent manner within the concentrations investigated in this study, and that segment-specific differences exist regarding the enhancing effect. One reason why the lowest absorption was observed in the colon may be due to the fact that the surface area for absorption is smaller than that in other segments. ${ }^{19)}$

Influence of Enzymatic Degradation of PLA on the Intestinal Absorption In order to investigate the influence of the enzymatic degradation of PLA on the intestinal absorption, $100 \mu \mathrm{g} / \mathrm{mL}$ aprotinin was co-administered with $0.5 \%$ PLA (Fig. 1, Table 1). In the duodenum, jejunum and ileum, the plasma concentration of FD-4 was increased remarkably after the co-administration of aprotinin, and the $F_{0-4 \mathrm{~h}}$ was also higher than that when $0.5 \%$ PLA was administered without the trypsin inhibitor. In particular, the $T_{\max }$ also increased in the duodenum and jejunum compared with the coadministration of FD-4 and $0.5 \%$ PLA alone, thus indicating that the effect was prolonged by preventing the enzymatic degradation of PLA. Meanwhile, the $T_{\max }$ was not changed by co-administration of aprotinin in the ileum, indicating that the protease has a little influence on the enhancing effect of PLA, that is, only a little protease might exist in the ileum compared with the duodenum and jejunum. ${ }^{20)}$ The mucous layer seems to be removed incompletely in a well-established in situ closed-loop method. It has been reported that the pancreatic enzymes capable of degrading proteins resided in the mucous layer. $^{21,22)}$ Hence, the effect of PLA may also be influenced 

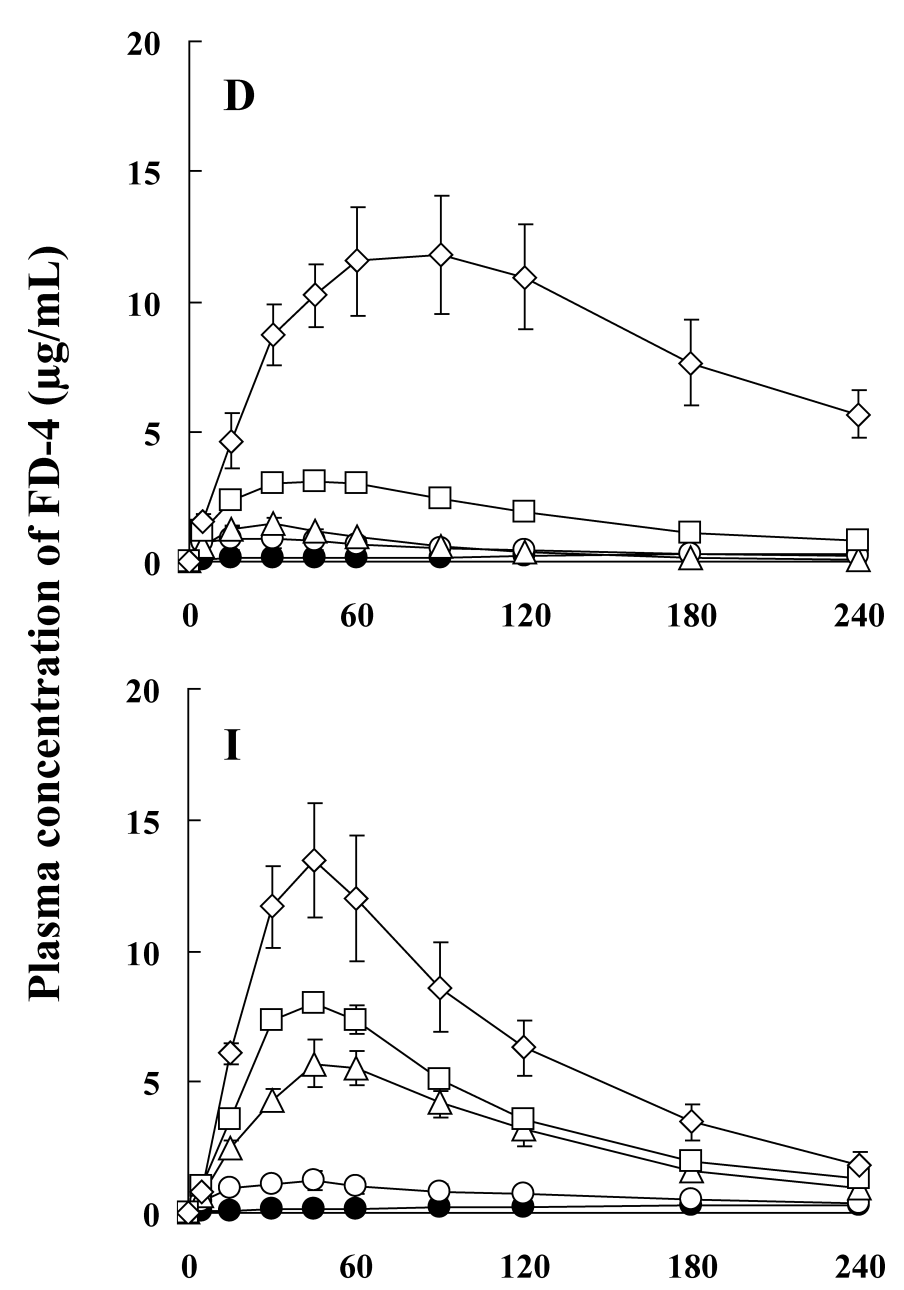

20
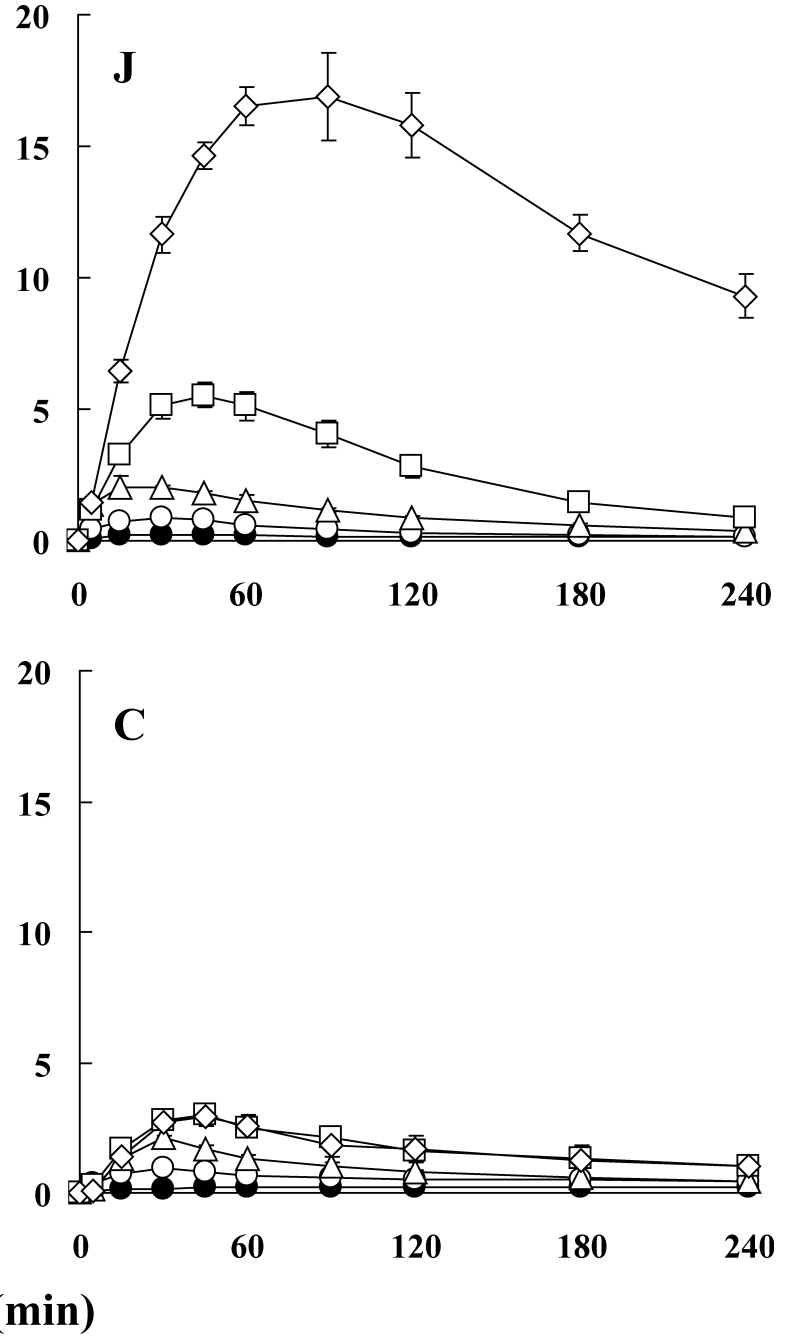

Fig. 1. The Plasma Concentrations of FD-4 Following Intra-intestinal Administration into the Closed-Loop in Rats

D: Duodenum, J: Jejunum, I: Ileum, C: Colon. •: Control (FD-4 only), ○: FD-4 with 0.05\% PLA, $\triangle$ : FD-4 with $0.25 \%$ PLA, $\square$ : FD-4 with 0.5\% PLA, $\diamond$ : FD-4 with $0.5 \%$ PLA and $100 \mu \mathrm{g} / \mathrm{mL}$ aprotinin. Each data point represents the mean and standard error $(n=3-7)$.

by the enzymes in the mucous layer. ${ }^{20)}$ The highest $F_{0-4 \mathrm{~h}}$ was obtained in the jejunum when aprotinin was co-administered with PLA. In contrast, the absorption of FD-4 was not observed to increase after the co-administering aprotinin in the colon, and the $F_{0-4 \mathrm{~h}}$ was almost equal to that when only $0.5 \%$ PLA was combined with FD-4. In addition, when $100 \mu \mathrm{g} /$ $\mathrm{mL}$ aprotinin alone was co-administered with FD-4 to each segment, the plasma concentrations of FD-4 were similar to those in the control group (FD-4 only) (data not shown). These findings corresponded with those of a previous study that reported the relationship between the enzymatic degradation of PLA and the enhancing effect on the nasal absorption of FD-4. ${ }^{4)}$ These results suggest that the effect of PLA on the absorption of FD-4 is affected by the enzymatic degradation of PLA in the small intestine, but that there is little influence of the enzymatic degradation on the absorption enhancement by PLA in the colon. Therefore, the differences in the enhancing effects of PLA on the absorption of FD-4 in the small intestine may be due to the amount of secreted enzyme. In addition, these differences may also be affected by the specific role of the duodenum as the first segment that encounters the acidic gastric contents. ${ }^{16)}$

Expression of Occludin in Different Intestinal Segments
Figure 2 shows the expression of occludin in the epithelium of the different rat intestinal segments. The occludin expression levels were almost identical in the duodenum, jejunum and ileum, but in colon, the level was higher than that in the other intestinal segments. Markov et al. previously compared the epithelial barrier properties with the presence of TJ proteins in rat intestinal segments by combining impedance spectroscopy and immunodetection. They demonstrated that the colon showed the highest epithelial resistance, followed by the duodenum, jejunum, and ileum, and also that the colon had the highest expression levels of occludin and claudins 1 , $3,4,5$, and 8 , which increased the epithelial barrier properties. ${ }^{16)}$ Their findings support the results of our present study. These results indicate that the paracellular barrier function in the colon epithelium is higher than that in other intestinal segments. Therefore, the smaller enhancing effect of PLA on the absorption of FD-4 in the colon may be related to the higher expression level of occludin, in addition to the smaller surface area for absorption in comparison to the other segments. In general, it has been reported that absorption enhancing effects of various absorption enhancers, such as sodium caprate, $n$-dodecyl- $\beta$-D-maltopyranoside and nitric oxide donors, are greater in the large intestine than those in the small intestinal 
Table 1. Pharmacokinetic Parameters of FD-4 Following Intra-intestinal Administration into the Closed-Loop in Rats

\begin{tabular}{|c|c|c|c|c|c|}
\hline Intestinal segment & FD-4 dose $(20 \mathrm{mg} / \mathrm{kg})$ & $C_{\max }(\mu \mathrm{g} / \mathrm{mL})$ & $T_{\max }(\min )$ & $A U C_{0-4 \mathrm{~h}}(\mu \mathrm{g} / \mathrm{mL} \cdot \min )$ & $F_{0-4 \mathrm{~h}}^{a)}(\%)$ \\
\hline \multirow[t]{5}{*}{ Duodenum } & Control & $0.29 \pm 0.13$ & $98.8 \pm 47.2$ & $49.8 \pm 23.8$ & 1.49 \\
\hline & $0.05 \%$ PLA & $0.97 \pm 0.39$ & $30.0 \pm 15.0$ & $117.7 \pm 42.4$ & 3.16 \\
\hline & $0.25 \%$ PLA & $1.46 \pm 0.26^{*}$ & $30.0 \pm 0.0$ & $125.8 \pm 8.3^{*}$ & 3.12 \\
\hline & $0.5 \%$ PLA & $3.26 \pm 0.28 * *$ & $38.6 \pm 4.5$ & $444.1 \pm 33.8 * *$ & 11.74 \\
\hline & $0.5 \%$ PLA $+100 \mu \mathrm{g} / \mathrm{mL}$ aprotinin & $12.17 \pm 1.93 *$ & $75.0 \pm 15.0$ & $2086.6 \pm 374.9 *$ & 57.31 \\
\hline \multirow[t]{5}{*}{ Jejunum } & Control & $0.36 \pm 0.07$ & $102.0 \pm 45.3$ & $39.6 \pm 8.9$ & 1.12 \\
\hline & $0.05 \%$ PLA & $0.90 \pm 0.23$ & $40.0 \pm 4.3$ & $93.8 \pm 13.7$ & 2.43 \\
\hline & $0.25 \%$ PLA & $2.24 \pm 0.25^{*}$ & $20.0 \pm 5.0$ & $245.8 \pm 27.5^{*}$ & 6.42 \\
\hline & $0.5 \%$ PLA & $5.56 \pm 0.50 * *$ & $42.9 \pm 2.0$ & $687.3 \pm 84.4 * *$ & 17.65 \\
\hline & $0.5 \% \mathrm{PLA}+100 \mu \mathrm{g} / \mathrm{mL}$ aprotinin & $17.44 \pm 1.27 * *$ & $80.0 \pm 10.0$ & $3054.1 \pm 196.5 * *$ & 84.90 \\
\hline \multirow[t]{5}{*}{ Ileum } & Control & $0.37 \pm 0.08$ & $162.5 \pm 36.0$ & $55.7 \pm 14.8$ & 1.70 \\
\hline & $0.05 \%$ PLA & $1.36 \pm 0.30$ & $30.0 \pm 7.5$ & $168.7 \pm 48.8$ & 4.50 \\
\hline & $0.25 \%$ PLA & $5.95 \pm 0.88 *$ & $50.0 \pm 5.0$ & $704.6 \pm 54.7 * *$ & 18.13 \\
\hline & $0.5 \%$ PLA & $8.06 \pm 0.40^{* *}$ & $47.5 \pm 4.6$ & $920.2 \pm 51.7 * *$ & 23.71 \\
\hline & $0.5 \% \mathrm{PLA}+100 \mu \mathrm{g} / \mathrm{mL}$ aprotinin & $13.44 \pm 2.16^{*}$ & $45.0 \pm 0.0$ & $1533.5 \pm 262.1 *$ & 39.12 \\
\hline \multirow[t]{5}{*}{ Colon } & Control & $0.32 \pm 0.04$ & $125.0 \pm 39.2$ & $48.9 \pm 9.3$ & 1.45 \\
\hline & $0.05 \%$ PLA & $0.96 \pm 0.14^{*}$ & $30.0 \pm 0.0$ & $137.2 \pm 21.3^{*}$ & 3.81 \\
\hline & $0.25 \%$ PLA & $2.09 \pm 0.09 * *$ & $30.0 \pm 0.0$ & $219.8 \pm 24.6^{*}$ & 5.83 \\
\hline & $0.5 \%$ PLA & $3.15 \pm 0.46^{* *}$ & $42.0 \pm 5.6$ & $410.5 \pm 57.4 * *$ & 11.08 \\
\hline & $0.5 \%$ PLA $+100 \mu \mathrm{g} / \mathrm{mL}$ aprotinin & $2.99 \pm 0.25 * *$ & $45.0 \pm 8.7$ & $394.1 \pm 95.1$ & 10.66 \\
\hline
\end{tabular}

a) $F_{0-4 \mathrm{~h}}$ was calculated from the amount of absorption obtained by means of a deconvolution method. $* p<0.05, * * p<0.01$ compared with control. Each data represents the mean or mean and standard error $(n=3-7)$.

a)

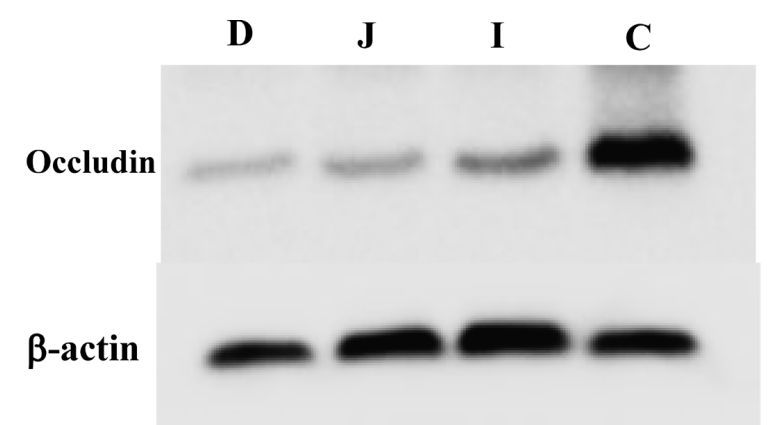

b)

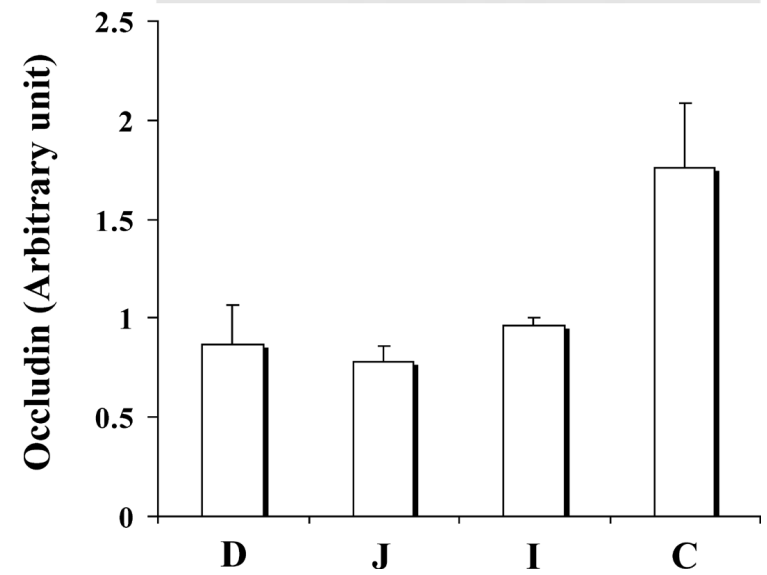

Fig. 2. The Expression of Occludin in the Epithelium of the Rat Intestinal Segments

a) Occludin and $\beta$-actin were detected by a Western blot analysis. b) The densitometry ratios representing the occludin expression in the epithelium. D: Duodenum, J: Jejunum, I: Ileum, C: Colon. Each data column represents the mean and standard error $(n=9)$.

regions. ${ }^{12,13)}$ The absorption enhancing mechanism of PLA might differ from that of these enhancers. In the future, an evaluation of the expression of other TJ proteins, such as claudins and ZO-1, in each intestinal segment will be needed to clarify the segment-specific differences regarding the effect of PLA on the intestinal absorption, and to develop a more effective transmucosal drug delivery system.

\section{CONCLUSION}

In conclusion, PLA enhanced the absorption of a hydrophilic macromolecule across the intestinal mucosa in rats. The enhancing effect of PLA was found to be dependent on its concentration within the concentrations investigated in this study. However, there were also segment-specific differences in the effect. These differences seem to be related to the surface area for absorption, the enzymatic degradation of PLA, and the expression of TJ proteins, such as occludin, in each intestinal segment.

\section{REFERENCES}

1) Natsume H, Iwata $S$, Ohtake $K$, Miyamoto M, Yamaguchi M, Hosoya K, Kobayashi D, Sugibayashi K, Morimoto Y. Screening of cationic compounds as an absorption enhancer for nasal drug delivery. Int. J. Pharm., 185, 1-12 (1999).

2) Miyamoto $M$, Natsume $H$, Iwata $S$, Ohtake $K$, Yamaguchi $M$, Kobayashi D, Sugibayashi K, Yamashina M, Morimoto Y. Improved nasal absorption of drugs using poly-L-arginine: effects of concentration and molecular weight of poly-L-arginine on the nasal absorption of fluorescein isothiocyanate-dextran in rats. Eur. J. Pharm. Biopharm., 52, 21-30 (2001).

3) Miyamoto M, Natsume H, Satoh I, Ohtake K, Yamaguchi M, Kobayashi D, Sugibayashi K, Morimoto Y. Effect of poly-L-arginine on the nasal absorption of FITC-dextran of different molecular weights and recombinant human granulocyte colony-stimulating factor (rhG-CSF) in rats. Int. J. Pharm., 226, 127-138 (2001).

4) Ohtake $K$, Natsume H, Ueda H, Morimoto Y. Analysis of transient and reversible effects of poly-L-arginine on the in vivo nasal absorption of FITC-dextran in rats. J. Control. Release, 82, 263-275 (2002). 
5) Ohtake K, Maeno T, Ueda H, Natsume H, Morimoto Y. Poly-Larginine predominantly increases the paracellular permeability of hydrophilic macromolecules across rabbit nasal epithelium in vitro. Pharm. Res., 20, 153-160 (2003).

6) Ohtake K, Maeno T, Ueda H, Ogihara M, Natsume H, Morimoto Y. Poly-L-arginine enhances paracellular permeability via serine/ threonine phosphorylation of ZO-1 and tyrosine dephosphorylation of occludin in rabbit nasal epithelium. Pharm. Res., 20, 1838-1845 (2003).

7) Nemoto E, Takahashi H, Kobayashi D, Ueda H, Morimoto Y. Effects of poly-L-arginine on the permeation of hydrophilic compounds through surface ocular tissues. Biol. Pharm. Bull., 29, 155-160 (2006).

8) Nemoto E, Ueda H, Akimoto M, Natsume H, Morimoto Y. Ability of poly-L-arginine to enhance drug absorption into aqueous humor and vitreous body after instillation in rabbits. Biol. Pharm. Bull., 30, 1768-1772 (2007).

9) Selg E, Acevedo F, Nybom R, Blomgren B, Ryrfeldt A, Gerde P. Delivering horseradish peroxidase as a respirable powder to the isolated, perfused, and ventilated lung of the rat: the pulmonary disposition of an inhaled model biopharmaceutical. J. Aerosol. Med. Pulm. Drug Deliv., 23, 273-284 (2010).

10) Yamaki T, Ohtake K, Ichikawa K, Uchida M, Uchida H, Ohshima S, Juni K, Kobayashi J, Morimoto Y, Natsume H. Poly-L-arginineinduced internalization of TJ proteins increases the paracellular permeability of the Caco-2 cell monolayer to hydrophilic macromolecules. Biol. Pharm. Bull., 36, 432-441 (2013).

11) Lee VHL, Yamamoto A. Penetration and enzymatic barriers to peptide and protein absorption. Adv. Drug Deliv. Rev., 4, 171-207 (1989).

12) Fetih G, Lindberg S, Itoh K, Okada N, Fujita T, Habib F, Artersson $\mathrm{P}$, Attia M, Yamamoto A. Improvement of absorption enhancing effects of $n$-dodecyl- $\beta$-D-maltopyranoside by its colon-specific delivery using chitosan capsules. Int. J. Pharm., 293, 127-135 (2005).

13) Fetih G, Habib F, Okada N, Fujita T, Attia M, Yamamoto A. Nitric oxide donors can enhance the intestinal transport and absorption of insulin and $\left[\mathrm{Asu}^{1,7}\right]$-eel calcitonin in rats. J. Control. Release, 106, 287-297 (2005).

14) Yamamoto A, Taniguchi T, Rikyuu K, Tsuji T, Fujita T, Murakami M, Muranishi S. Effects of various protease inhibitors on the intestinal absorption and degradation of insulin in rats. Pharm. Res., 11, 1496-1500 (1994).

15) Tanaka K, Fujita T, Yamamoto Y, Murakami M, Yamamoto A, Muranishi S. Enhancement of intestinal transport of thyrotropinreleasing hormone via a carrier-mediated transport system by chemical modification with lauric acid. Biochim. Biophys. Acta, 1283, 119-126 (1996).

16) Markov AG, Veshnyakova A, Fromm M, Amasheh M, Amasheh S. Segmental expression of claudin proteins correlates with tight junction barrier properties in rat intestine. J. Comp. Physiol. B, 180, 591-598 (2010).

17) Sugita Y, Takao K, Toyama Y, Shirahata A. Enhancement of intestinal absorption of macromolecules by spermine in rats. Amino Acids, 33, 253-260 (2007).

18) Yamaoka K, Tanigawara $Y$, Nakagawa T, Uno T. A pharmacokinetic analysis program (multi) for microcomputer. J. Pharmacobiodyn., 4, 879-885 (1981)

19) Wilson TH. Intestinal Absorption. Saunders, Philadelphia and London (1962).

20) Aoki Y, Morishita M, Asai K, Akikusa B, Hosoda S, Takayama K. Region-dependent role of the mucous/glycocalyx layers in insulin permeation across rat small intestinal membrane. Pharm. Res., 22, 1854-1862 (2005).

21) Ugolev AM, Smirnova LF, Iezuitova NN, Timofeeva NM, Mityushova NM, Egorova VV, Parshkov EM. Distribution of some adsorbed and intrinsic enzymes between the mucosal cells of the rat small intestine and the apical glycocalyx separated from them. FEBS Lett., 104, 35-38 (1979).

22) Goldberg DM, Campbell R, Roy AD. Binding of trypsin and chymotrypsin by human intestinal mucosa. Biochim. Biophys. Acta, 167, 613-615 (1968). 